\title{
DESIGN OF POROUS TI-6AL-4V ALLOY STRUCTURES FOR BIOIMPLANTS
}

\author{
S. RAMBABU \\ Department of Mechanical Engineering, Vignan's Institute of Information Technology, Andhra Pradesh, India
}

\begin{abstract}
This paper presents the efforts made in studying the elastic modulus of porous Ti-6Al-4V alloy structures for biomedical load-bearing applications. The major issues concerning with the metallic implants is the mismatch of elastic modulus between the cortical bone and the metallic implant which resulted in stress shielding and cause to disassociation of the implant with the surrounding bone. In addition to this, fixation of the implant to the bone is crucial in the implant functioning. In present work, the elastic modulus of porousTi-6Al-4V alloy structure with different porosity ranging from 10 vol.\% to 50 vol.\% were estimated using the Nielsen's relationship. From the results, it is found that the porousTi-6Al$4 \mathrm{~V}$ alloy structure with the porosity of 50 vol. \% possesses the elastic modulus about 24.19GPa which is close to the human cortical bone. Fabrication of porous implant structures with the designed porosity is difficult with the conventional manufacturing techniques. The additive manufacturing technology capable of produce the porous implants with the designed porosity. We have demonstrated the proof of tailoring the elastic modulus of metallic implants with the designed porosity and minimise the mismatch of elastic modulus between theTi-6Al-4V alloy structure and the human cortical bone.

KEYWORDS: Porosity, Ti-6Al-4V Alloy, Elastic Modulus, Additive Manufacturing
\end{abstract}

Received: Jun 08, 2020; Accepted: Jun 28, 2020; Published: Aug 18, 2020; Paper Id.: IJMPERDJUN2020788

\section{INTRODUCTION}

The need for load-bearing bioimplants specifically orthopaedic and dental implants have significantly increased in the last two decades since the patients are expecting treatments to replace, support or enhance a failing bone structuresto maintain their daily activities and quality of life. The bioimplants are made of biosynthetic materials, used for structural reinforcements inside the human body for load-bearing applications including hip implants, knee implants, dental implants, screws and plates for spinal surgeries. The biomaterials should possess the good biocompatibility, desirable mechanical properties, wear resistance, and osseointegration for the effective functioning in the human body. Among the various biocompatible metals, cobalt-chromium (Co-Cr) alloys, stainless steel (316L), nickel titanium (NiTi) alloys and titanium alloys extensively used for load-bearing implant applications (Munro and McGrath, 2015;Kuncickáet al., 2017).The value of the biomaterials market was about US\$134.3 billion in 2017 worldwide (Yuan et al., 2019). However, the major problems in biomaterials design is the significant mismatch of elastic modulus between the cortical bone and the implant, which results in stress shielding phenomenon and leads to implant loosening from the surrounding bone(Krishna et al., 2007; Asgari and Mohammadi, 2018). Typically, the elastic modulus of cortical bone is ranging from 10 to 30 GPawhereas the metal implants made of Co-Cr alloys, 316L stainless steel, Ti-6Al-4V alloy exhibits the highest elastic modulus about 248GPa, 210GPa, and $110 \mathrm{GPa}$, respectively. Moreover, the interfacial bonding between the living bone and the metallic implant is less due to bio-inert nature of metals, which cause to inadequate bone formation and in growth. These problems can be minimise by developing the implant structures with the designed porosity. 
Recently, there has been increasing interest in the development of porous implant structures, because the porous structure can provide the space for new bone tissue in growth and body fluid circulation, and possess adequate mechanical properties. The porosity is the percentage of void space in a solid structure, and it influences on the biocompatibility and mechanical properties including yield strength, elastic modulus, fracture toughness and fatigue strength of the implants (Al-Ketan et al., 2018). Several types of conventional manufacturing techniques employed to fabricate the porous implant structures (Ryan et al., 2006), including powder metallurgy techniques (Asaokaet al.,1985; Oh et al., 2003), liquid foaming method (Chen et al., 2009), solid-state foaming, microsphere sintering (Reig et al., 2012), space holder technique (Torres et al., 2012), precision casting (Müller et al., 2012), directional freeze casting technique(Mao et al., 2018), extrusion process (Meredith and Mallick, 2014), replication and plasma spraying technique (Ryan et al., 2006). However, fabrication of the complex porous implant structures with controlled porosity shape, size and volume is difficult with conventional manufacturing techniques. Hence, advanced manufacturing techniques are essential to fabricate the complex implant structures with designed macro and micro porosity.

The additives manufacturing (AM) is one of such technology, capable of novel design and manufacture the porous metallic implant structures with complex designed porosity. Additive manufacturing is a process that produces the parts by adding the material together, typically layer-by-layer, based on the three dimensional (3D) computer aided design (CAD) data. The AM technology allows complex designs and also impact on the manufacturing procedures in the biomedical engineering. Due to the improvement in additive manufacturing technology and the availability of various materials, the AM technology is using to produce porous orthopaedic implant structures and scaffolds (Krishna et al., 2007;Bandyopadhyayet al., 2010).The several types of AM techniques are available including selective laser sintering (SLS), selective laser melting (SLM),laser-engineered net shaping (LENS) and electron beam melting (EBM)for development of the intricate geometries, functionally graded and porous metallic implant structures based on the patient requirements.

The fabrication of load-bearing metallic implants with the controlled porosity attracted the attention of researchers and consumers because of their superior biocompatibility and adequate mechanical properties (Yuan et al., 2019; Savio et al., 2019; Torres et al., 2012). Robertson et al., (1976) studied the fixation of porous implant of Stellite-21 ${ }^{\text {TM }}$ (cobaltchromium alloy) with the femora bone of adult mongrel dog, and the shear strength of the bone-implant interface. The experimental results showed that the strong bonding between the bone and the porous-surfaced implant was established by in growth of the new bone into the porous structure. The shear strength at the bone-implant interface increases with pore size and time of healing. The porous titanium dental implants produced by powder sintering technique possesses the adequate mechanical properties such as compressive strength and fatigue strength, better biomechanical compatibility than the high elastic modulus materials such as ceramics (Asaoka et al., 1985). Krishna et al., (2007) developed the porous commercially pure (CP) titanium implant structures with three-dimensionally interconnected porosities using laserengineered net shaping process. They found that the elastic modulus of porous titanium samples with porosity ranging from 35 vol.\% to 42 vol.\% close to that of human cortical bone. Bandyopadhyay et al., (2010) studied the elastic modulus and In vivo behaviour of the porous Ti-6Al-4V alloy structures fabricated using the laser-engineered net shaping process, demonstrated tailoring the elastic modulus of porous implant structures with designed porosity. In vivo studies showed that the porous Ti-6Al-4V alloy samples in rats for 16 weeks significantly enhanced the tissue in growth through interconnected pores. 
In recent years, the porous structures with three dimensional triply periodic minimal surface (TPMS) based cellular structures are drawing researchers attention, because these structures possesses superior biomimicry features for cell activities such as cell attachment, penetration and proliferation. Moreover, TPMS cellular structures exhibits superior mechanical properties (Wang et al., 2016;Zhang et al., 2017;Tan et al., 2017;Bobbert et al., 2017;Afsher et al. 2018; AlKetan et al., 2018) designed and fabricated the various periodic cellular structures with strut based, skeletal TPMS and sheet TPMS based diamond, gyroid and primitive structures. The experimental results showed that the sheet TPMS based diamond cellular structure exhibits the superior mechanical performance compared to other cellular structures.

From the above, it is clear that the porous structures possesses superior biocompatibility, lower elastic modulus and adequate compressive strength, and fatigue properties which results in promising solution for orthopaedic implants. However, the increase in porosity reduces the yield strength of the implant. Hence, balancing the biocompatibility and mechanical properties is essential in the development of a superior load-bearing metallic implant structures. Due to the unique capabilities of additive manufacturing technology, make it possible to manufacture the parts with designed porosity. Hence, this work is envisaged to design the porous Ti-6Al-4V alloy structures for additive manufacturing, and predict the elastic modulus by using the Nielsen's relationship.

\section{MATERIALS AND METHODS}

Among the available metallic biomaterials for load-bearing biomedical applications, titanium and its alloys, specifically Ti6Al-4V alloy recognised as the significant biomaterial for load-bearing applications due to their superior biocompatibility, desirable mechanical properties, resistance to corrosion, low density and high strength-to-weight ratio. However, the elastic modulus of Ti-6Al-4V is about $110 \mathrm{GPa}$ whereas the human cortical bone having the elastic modulus about $20 \mathrm{GPa}$. The elastic modulus of various bioimplant materials and human cortical bone was presented in the Figure 1. From the elastic modulus values presented in the Figure 1, it is clear that the significant mismatch between the metallic implant materials and the human cortical bonemay resulted in disassociation of the implant with the human bone

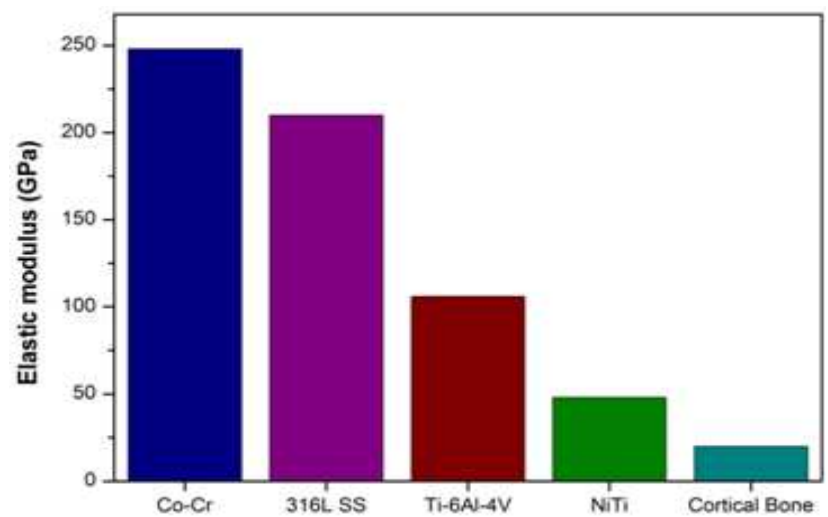

Figure 1: Elastic Modulus of Various Metallic Biomaterials and Cortical Bone.

To attain the elastic modulus of implant close to the human cortical bone and increase the ingrowth of bone into the implant structure, the implant should be fabricate with the appropriate porous structure.Typically, the first step involved in additive manufacturing of the porous Ti-6Al-4V alloy structure is design the 3D CAD model of the part to be produced, convert it into standard tessellation language (STL) file format and transfer to the 3D printing machine. Figure 2 presents the important steps involved in the additive manufacturing of the porous Ti-6Al-4V structures using direct metal laser sintering (DMLS) process. 


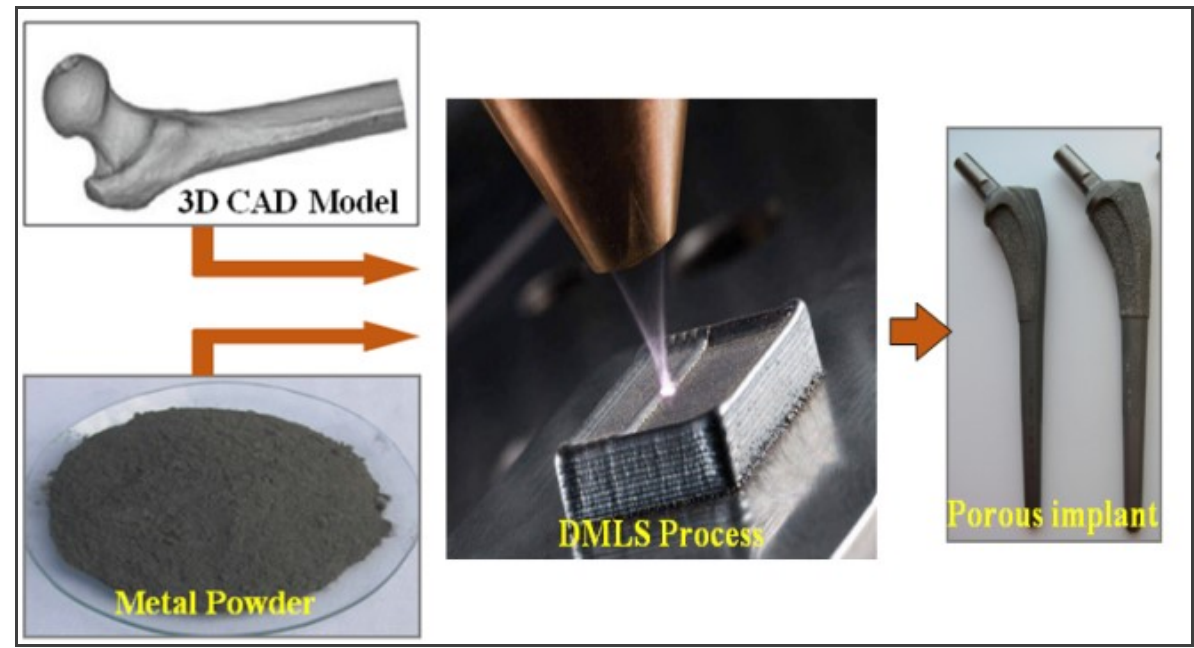

Figure 2: Direct Metal Laser Sintering of Porous Ti-6Al-4V Alloy Implants.

Direct metal laser sintering is one of the promising technologies of additive manufacturing of various metals and its alloys. In DMLS, a micron-size thin layer of a powder is spread on the build platform and high power laser melts the powder layer at the specified points defined by a three dimensional CAD model. After melting of thin layer of powder, the platform moves down wards and then a new layer of powder spread on it. The same procedure is repeated up to the final part is built. This process allows fabricating the porous structures with the designed macro and micro porosities.

\section{Design of Porous Ti-6Al-4V Alloy Structures}

The important factor for design of various porous structures is the relationship between the porosity volume and the elastic modulus. The simple cube is developed with different porosities ranging from 10 vol.\% to 50 vol.\% using square holes to study the influence of porosity volume on the elastic modulus. Figure 3 (a-c) presents the porous cube structures with 10 vol.\%, 30 vol.\% and 50 vol.\% of porosity.

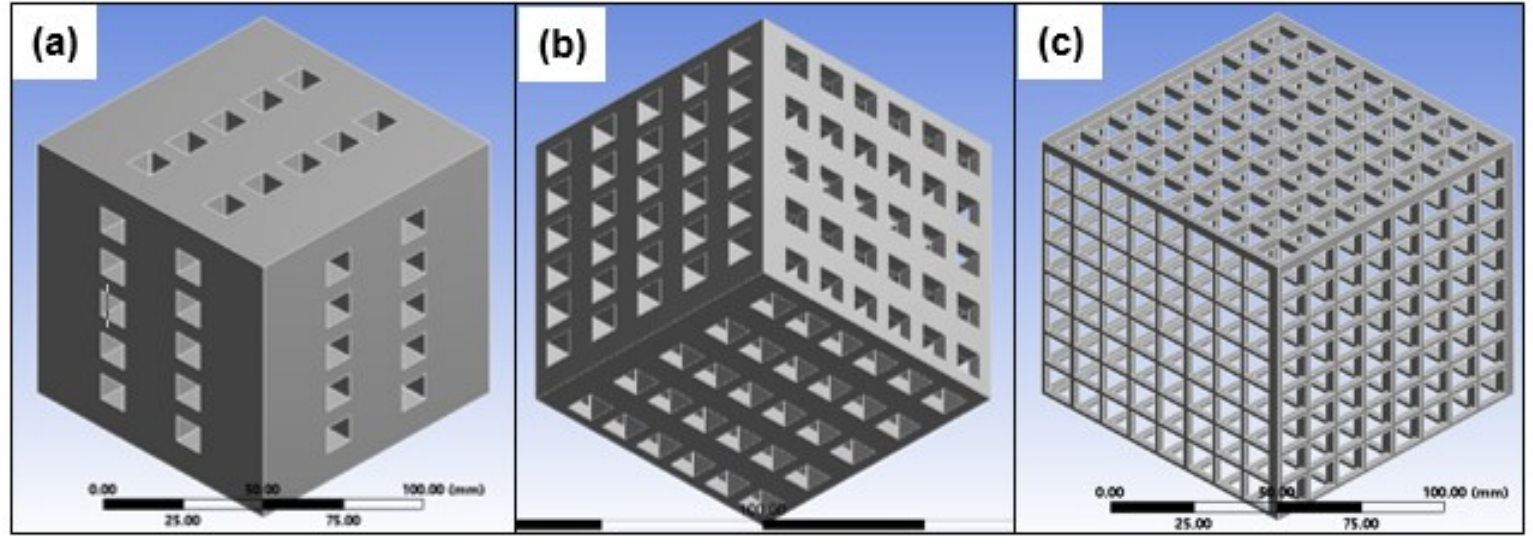

Figure 3: Design of Cube Structures with (a) 10 vol.\%, (b) 30 vol.\% and (c) 50 vol.\% of Porosity.

In this work, the elastic modulus of the porous Ti-6Al-4V alloy structures ranging from 10 vol. $\%$ to 50 vol. $\%$ were estimated using the Nielsen's relationship (Nielsen 1990). 


$$
\mathrm{E}=\frac{\mathrm{E}_{m}(1-v)^{2}}{1+\left(\frac{1}{\rho}-1\right) v}
$$

Where $\mathrm{E}$ is the elastic modulus of porous material, $\mathrm{E}_{\mathrm{m}}$ is the elastic modulus of fully dense material, $v$ is the volume fraction of porosity and $\rho$ is the geometry factor based on the pore shape.

$$
\rho=\frac{4 \times \pi \times \text { area }^{2}}{\text { Perimeter }}
$$

\section{RESULTS AND DISCUSSIONS}

The simple cube structures with porosities ranging from 10 vol.\% to 50 vol.\% were designed using the 3D CAD model. The elastic modulus of porous Ti-6Al-4V alloy structures estimated by using the Nielsen's relationship and presented in the Table 1. From the results presented in Table 1, it is clear that the elastic modulus of Ti-6Al-4V alloy decreases with increase the volume of porosity. The Ti-6Al-4V alloy without porosity possesses $110 \mathrm{GPa}$ and gradually deceased from $86.73 \mathrm{GPa}$ to $24.19 \mathrm{GPa}$ while increasing the porosity volume from 10 vol. $\%$ to 50 vol. $\%$, respectively. From these results, it is found that the elastic modulus of the porousTi-6Al-4V alloy structure with 50 vol. $\%$ is very close to the elastic modulus of the cortical bone, shown in Figure 4. The results demonstrating the proof of tailoring the elastic modulus of metallic implant structures. In addition to this, the porous implant structures can enhance the bone in growth into the implant which leads to long-term stability.

Table 1: Elastic Modulus of Porous Ti-6Al-4V Alloy Structures

\begin{tabular}{|c|c|c|}
\hline S. No. & Porosity vol.\% & Elastic modulus (GPa) \\
\hline 1 & 10 & 86.73 \\
\hline 2 & 20 & 66.75 \\
\hline 3 & 30 & 49.81 \\
\hline 4 & 40 & 35.69 \\
\hline 5 & 50 & 24.19 \\
\hline
\end{tabular}

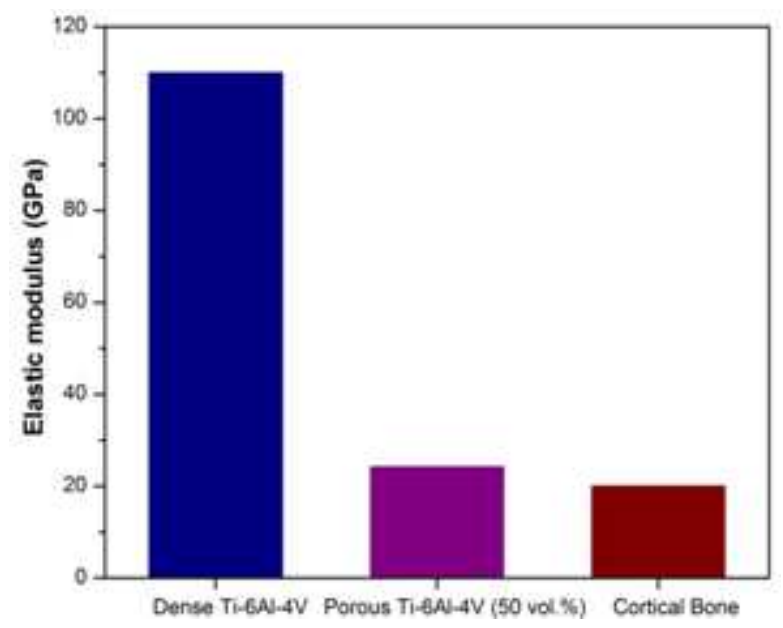

Figure 4: Elastic Modulus of Dense, Porous (50 vol.\%) Ti-6Al-4V Alloy and Cortical Bone. 


\section{CONCLUSIONS}

This paper presents the design of porous Ti-6Al-4V alloy structures for load-bearing biomedical applications. The Neilsen's relationship was employed to determine the elastic modulus of the porous Ti-6Al-4V alloy structures with porosity ranging from $10 \mathrm{vol} \%$ to $50 \mathrm{vol} \%$. From the results, it is found that theTi-6Al-4V alloy structure with the porosity of $50 \mathrm{vol} . \%$ possess the elastic modulus of $24.19 \mathrm{GPa}$ which is close to the human cortical bone. From this work, it is clear that the elastic modulus of the implant can be tailored by designing the porosity. Moreover, the porous implant structures can enhance the fixation of implant to the bone. Future work includes the experimental validation of elastic modulus of the porous Ti-6Al-4V alloy structures with the present results.

\section{ACKNOWLEDGEMENTS}

This research received no specific grant from any funding agency in the public, commercial or not-for-profit sectors. No acknowledgements.

\section{REFERENCES}

1. Afshar M, PourkamaliAnaraki A and Montazerian H (2018) Compressive characteristics of radially graded porosity scaffolds architectured with minimal surfaces. Material Science \& Engineering C 92: 254-267.

2. Al-Ketan $O$, Rowshan $R$ and Abu Al-Rub RK (2018) Topology-mechanical property relationship of $3 D$ printed strut, skeletal, and sheet based periodic metallic cellular materials. Additive Manufacturing 19: 167-183.

3. Asaoka K, Kuwayama N, Okuno O et al. (1985) Mechanical properties and biomechanical compatibility of porous titanium for dental implants. Journal of biomedical materials research 19(6): 699-713.

4. Asgari H and Mohammadi M (2018) Microstructure and mechanical properties of stainless steel CX manufactured by Direct Metal Laser Sintering. Materials Science \& Engineering A709: 82-89.

5. Apparao, D., and M. V. Jagannadha Raju. "Experimental investigation on hardness, microstructure and surface roughness of maraging steel parts produced by direct metal laser sintering technique." Int J Mech Prod Eng Res Dev (IJMPERD) 8.2 (2018): 629-636.

6. Bandyopadhyay A, Espana F, Krishna V et al. (2010) Influence of porosity on mechanical properties and in vivo response of Ti6Al4V implants. ActaBiomaterialia6(4): 1640-1648.

7. Bobbert FSL, Lietaert K, Eftekhari AA et al. (2017) Additively manufactured metallic porous biomaterials based on minimal surfaces: A unique combination of topological, mechanical, and mass transport properties. ActaBiomaterialia53: 572-584.

8. Chen YJ, Feng B, Zhu YP et al. (2009) Fabrication of porous titanium implants with biomechanical compatibility. Materials Letters 63(30): 2659-2661.

9. Krishna BV, Bose S and Bandyopadhyay A (2007) Low stiffness porous Ti structures for load-bearing implants. Acta Biomaterialia 3(6): 997-1006.

10. Kuncická L, Kocich $R$ and Lowe TC (2017) Advances in metals and alloys for joint replacement. Progress in Materials Science 88:232-280. 
11. Mao M, Tang Y, Zhao K et al. (2018) Porous titanium scaffolds with aligned lamellar pore channels by directional freeze casting from aqueous- TiH2 slurries. Metals and Materials International 25(2): 508-515.

12. Meredith $J$ and Mallick KK (2014) High-strength scaffolds for bone regeneration. Bioinspired, Biomimetic and Nanobiomaterials4(1): 48-58.

13. Müller DW, Matz AM and Jost N (2012) Casting open porous Ti foam suitable for medical applications. Bioinspired, Biomimetic and Nanobiomaterials2(2): 76-83.

14. Munro NH and McGrath (2015) Advances in techniques and technologies for bone implants. Bioinspired, Biomimetic and Nanobiomaterials4(1): 26-36.

15. Nielsen LF (1990) Elasticity and damping of porous materials and impregnated materials. Journal of American Ceramic Society 67(2): 93-98.

16. Oh IH, Nomura N, Masahashi $N$ et al. (2003) Mechanical properties of porous titanium compacts prepared by powder sintering. ScriptaMaterialia49(12): 1197-1202.

17. Poonia, Hemant, and R. C. Chaudhary. "Mass transfer with chemical reaction effects on MHD free convective flow past an accelerated vertical plate embedded in a porous medium." Int. J. of Applied Mathematics \& Statistical Sciences 5 (2016).

18. Ramaswamy, $R$., et al. "Investigation of densification behaviour on yttrium oxide reinforced Ti-6Al-4V nanocomposite through powder metallurgy." Int J Mech Prod Eng Res Dev 8.2 (2018): 433-442.

19. Ramadurai, K., V. Sathan Raj, and R. Vinoth. "Analysis of wear and sem characteristics on ferrous composites through powder metallurgy method." International Journal of Mechanical and Production Engineering Research and Development (IJMPERD 8.4 (2018): 609-614.

20. Reig L, Amigó V, Busquets DJ et al. (2012) Development of porous Ti6Al4V samples by microsphere sintering. Journal of Materials Processing Technology 212(1): 3-7.

21. Robertson DM, Pierre L and Chahal $R$ (1976) Preliminary observations of bone ingrowth into porous materials. Journal of biomedical materials research10(3): 335-344.

22. Ryan G, Pandit A and Apatsidis DP (2006) Fabrication methods of porous metals for use in orthopaedic applications. Biomaterials 27: 2651-2670.

23. Savio G, Rosso S, Curtarello A et al. (2019) Implications of modeling approaches on the fatigue behavior of cellular solids. Additive Manufacturing 25: 50-58.

24. Tan XP, Tan YJ, Chow CSL et al. (2017) Metallic powder-bed based 3D printing of cellular scaffolds for orthopaedic implants: A state-of-the-art review on manufacturing, topological design, mechanical properties and biocompatibility. Materials Science and Engineering C 76: 1328-1343.

25. Torres Y, Rodríguez JA, Arias S et al. (2012) Processing, characterization and biological testing of porous titanium obtained by space-holder technique. Journal of Materials Science 47(18): 6565-6576.

26. Wang X, Xu S, Zhou $S$ et al. (2016) Topological design and additive manufacturing of porous metals for bone scaffolds and orthopaedic implants : A review. Biomaterials 83: 127-141.

27. Yuan L, Ding S and Wen C (2019) Additive manufacturing technology for porous metal implant applications and triple minimal surface structures: A review. Bioactive Materials 4(1): 56-70. 
28. Zhang XY, Fang $G$ and Zhou J (2017) Additively manufactured scaffolds for bone tissue engineering and the prediction of their mechanical behavior: A review. Materials 10: E 50. 